\title{
ISO Spectra of Planetary Nebulae
}

\author{
Kevin Volk and Sun Kwok \\ Dept. of Physics and Astronomy, University of Calgary, Calgary, \\ Canada T2N 1 N4
}

\section{Introduction}

It has been well known since the IRAS mission that dust emission represents a significant fraction of the energy output from PNe (Zhang \& Kwok 1991). Although the dust component in PNe was long thought to be due to the remnants of the envelopes of AGB stars (Kwok 1982), we now know that dust in PNe has a much richer chemical composition. In addition to amorphous silicates and $\mathrm{SiC}$ features commonly seen in AGB stars, PNe have been found to have strong aromatic infrared features (Russell et al. 1977), crystalline silicate features (Waters et al. 1997), and an unidentified emission feature at $30 \mu \mathrm{m}$ (Forrest et al. 1981). In this paper, we show the ISO spectra of a number of PNe illustrating the diverse dust chemistry in PNe.

\section{Results}

Figure 1 shows $I S O$ spectra of $4 \mathrm{PNe}$ as examples of the following classes of dust compositions.

- Aromatic hydrocarbons: many carbon-rich PNe show strong emission features at $3.3,6.2,7.7,8.6$, and $11.3 \mu \mathrm{m}$ characteristic of aromatic compounds. IRAS $21282+5050$ shows strong aromatic hydrocarbon bands (AHB) with very few emission lines.

- Aromatic hydrocarbon and crystalline silicates: Cn1-1 shows a strong continuum due to AHB and crystalline silicate bands. No emission lines are seen.

- Amorphous silicates: the 10 and $18 \mu \mathrm{m}$ amorphous silicates features, seen in over 3000 oxygen-rich AGB stars, are present in the spectra of PNe. The spectrum of H2-1 shows amorphous silicate features in emission.

- Peculiar: Mz-3 is a bipolar reflection nebula with a cool (32000 K) central star. With the exception of some crystalline silicate features, the dust continuum is of unknown origin and has a peculiar shape.

\section{Discussion}

While the general infrared continuum of PNe is due to dust (e.g. amorphous carbon and amorphous silicates) left over from the AGB, it is clear that new dust 

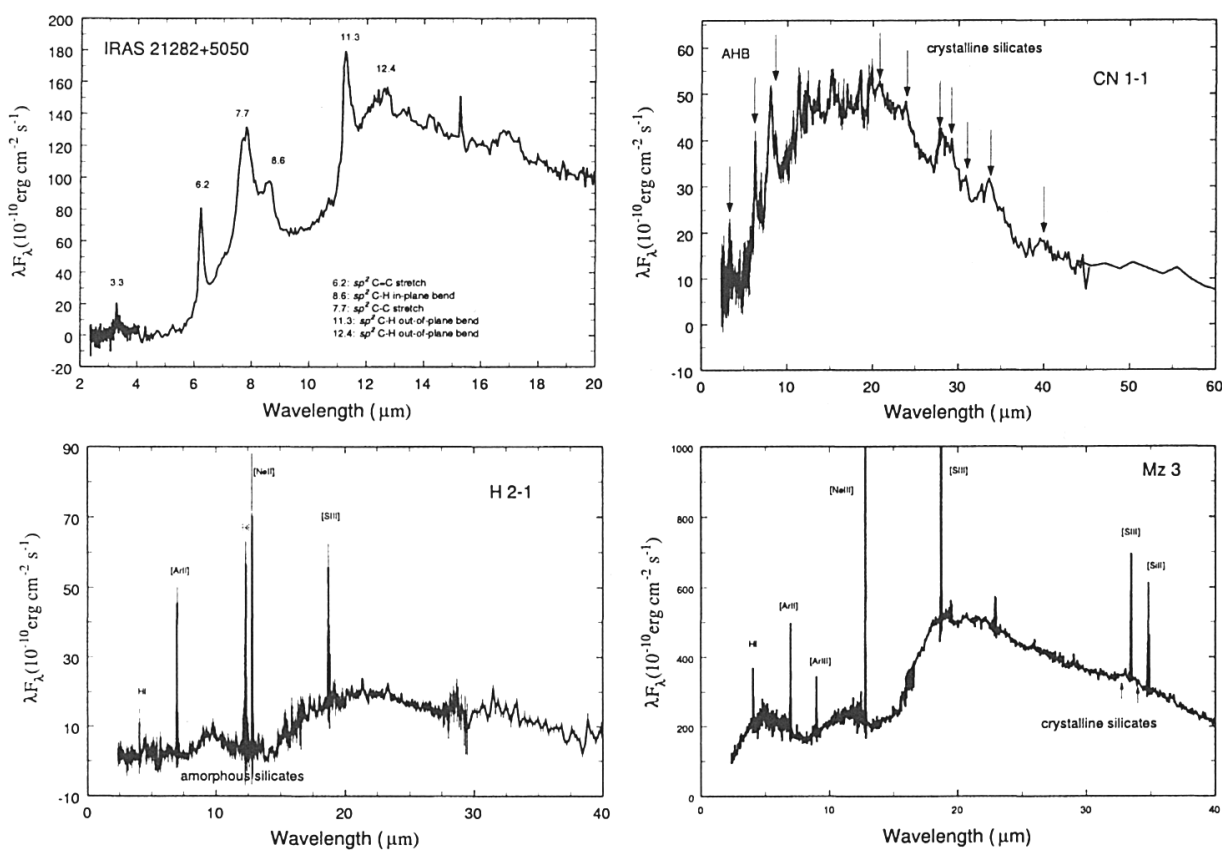

Figure 1. ISO spectra (clockwise from top left) of IRAS 21282+5050, Cn1-1, Mz-3 and H2-1, illustrating different dust compositions in PNe.

components (aromatic compounds and crystalline silicates) are formed beyond the AGB. Since the dynamical lifetime of the nebulae is only $\sim 10^{4} \mathrm{yr}$, the chemical processes forming these material must be extremely efficient (Kwok et al. 1999). The change in the level of the 8 and $12 \mu \mathrm{m}$ plateau features from $\mathrm{PPNe}$ to $\mathrm{PNe}$ also suggests that photochemistry plays a role in evolution of the dust component (Kwok et al. 2001).

\section{References}

Kwok, S. 1982, ApJ, 258, 280

Kwok, S., Volk, K., \& Hrivnak, B.J. 1999, A\&A, 350, L35

Kwok, S., Volk, K., \& Bernath, P. 2001, ApJ, 554, L87

Forrest, W.J., Houck, J.R., \& McCarthy, J.F. 1981, ApJ, 248, 195

Russell, R.W., Soifer, B.T., \& Wilner, S.P. 1977, 217, L149

Waters, L.B.F.M., et al. 1997, A\&A, 315, L361

Zhang, C.Y., \& Kwok, S. 1991, A\&A, 250, 179 